\title{
Pediatric Ambulatory ECMO
}

\author{
Don Hayes Jr. • Eric A. Lloyd • Andrew R. Yates • \\ Patrick I. McConnell • Mark Galantowicz • \\ Thomas J. Preston
}

Received: 4 July 2014/ Accepted: 1 September 2014/Published online: 10 September 2014

(C) Springer Science+Business Media New York 2014

To the Editor,

The current literature provides a modest experience regarding ambulating adult patients on venovenous extracorporeal membrane oxygenation (VV-ECMO), with some older teens also reported. However, there is no previous report of ambulation in a younger child.

We report the ambulation of an 8-year-old child on VVECMO who developed acute respiratory distress syndrome due to a medication reaction to trimethoprim/sulfamethoxazole. At his initial presentation, he had developed fever, cough, and dyspnea soon after starting the antimicrobial coverage for osteomyelitis. With worsening respiratory status and facial swelling, he presented to a community pediatric facility where he was found to have a pneumothorax and was intubated. Due to refractory hypercapnia, he was transferred to a quaternary pediatric facility for implementation of VV-ECMO through cannulation of the internal jugular with a bicaval dual-lumen catheter. With a prolonged course in the intensive care unit, he underwent tracheostomy. To avoid complications from immobilization, he was ambulated on VV-ECMO and underwent active participation of physical therapy including riding a hand and foot-pedaled tricycle (Figure).

D. Hayes Jr. $(\bowtie) \cdot$ E. A. Lloyd · A. R. Yates ·

P. I. McConnell · M. Galantowicz - T. J. Preston

Nationwide Children's Hospital, The Ohio State University,

700 Children's Drive, Columbus, OH 43205, USA

e-mail: hayes.705@osu.edu

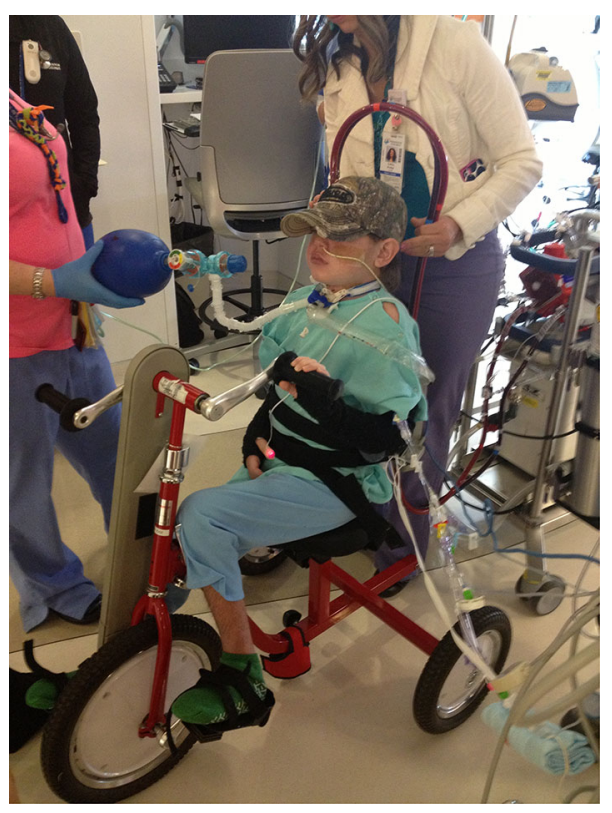

In a critical care setting especially with ECMO support, prolonged immobilization can result in skeletal muscle atrophy and loss of muscle fibers, which can result in the loss of muscle strength, decreased range of motion, and contractures. Moreover, this clinical situation can further be complicated by polyneuropathy and myopathy due to critical illness, which can further worsen muscle wasting and weakness. Based on our experience, ambulatory VVECMO can be safely performed in early school-age children while allowing them to improve muscle strength on invasive extracorporeal support.

Conflict of interest None. 